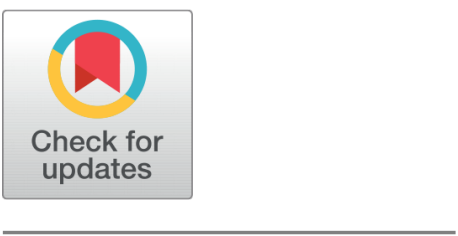

6 OPEN ACCESS

Received: 19-03-2020

Accepted: 07-05-2020

Published: 18-06-2020

Editor: Dr. Natarajan Gajendran

Citation: Calo AMVM, Barbosa JB, Dinagsao AV (2020) GrabTutor: A web and mobile application for tutor appointment system with GPS security feature. Indian Journal of Science and Technology 13(19): 1955-1964. https://doi.org/ 10.17485/IJST/v13i19.148996

* Corresponding author. Al-Monte Vince M Calo

Department of Information Technology, College of Information Technology and Computing University of Science and

Technology of Southern Philippines, C.M. Recto Avenue, Lapasan,

Cagayan de Oro City, 9000,

Philippines

vince.calo@ustp.edu.ph

Funding: None

Competing Interests: None

Copyright: (c) 2020 Calo, Barbosa, Dinagsao. This is an open access article distributed under the terms of the Creative Commons Attribution License, which permits unrestricted use, distribution, and reproduction in any medium, provided the original author and source are credited.

Published By Indian Society for Education and Environment (iSee)

\section{GrabTutor: A web and mobile application for tutor appointment system with GPS security feature}

\author{
Al-Monte Vince M Calo1* , Jocelyn B Barbosa1 , Amparo V Dinagsao1 \\ 1 Department of Information Technology, College of Information Technology and Computing \\ University of Science and Technology of Southern Philippines, C.M. Recto Avenue, Lapasan, \\ Cagayan de Oro City, 9000, Philippines
}

\section{Abstract}

Background/Objectives: Academic tutoring is sought to aid students academically, keep them in school, and eventually earn a degree aside from the learning they get from a typical classroom setting. Academic institutions somehow start programs for peer tutorial sessions among students, but matching of tutees with tutors takes time especially for complex and complicated topics. It even brings a security concern about where students are conducting tutorials. Hence, this study aims to design a tutor appointment system with location tracking feature to give students a more efficient and safer tutorial experience. Methods: GrabTutor is composed of a mobile and web application that communicates through a cloud server. The tutees find tutors through the mobile application. The system generates a unique barcode for each tutorial appointment. A barcode scanner is embedded in the mobile application accessible to the tutor. Once the tutor scans, the system will trigger the timer and sends GPS coordinates to the server to track the location. The location will be represented by a marker on the map of the moderator's web application. Findings: Based on the results of the usability tests, the system easily matched tutees to the right tutors. The GPS monitoring system was proven to be a helpful tool to ease the risk of students having to go somewhere else to meet up with a tutor. Novelty/Applications: The proposed system enables quick and efficient matching of tutors to tutees and integrates GPS information of students within the campus during the conduct of the tutorial to track their whereabouts for a more secure tutorial experience.

Keywords: Tutor appointment; location tracking; barcode; GPS; security

\section{Introduction}

While teachers remain to be the primary facilitator of learning in every level of education, peer tutoring among students of same age brackets have received positive reviews from educators all around the world as an intervention 
to support student learning outside the typical classroom setup. Studies have shown that peer tutorial is an effective strategy to practice academic skills with someone from the same age bracket ${ }^{(1,2)}$. With this, many online tutor appointment or scheduling applications have been coming out for the convenience of prospective tutors and tutees. Furthermore, many developers create mobile scheduling applications since almost everyone has already a smartphone and it makes it more convenient for people to make an appointment wherever they are and whenever they want to.

The academic support of schools provides tutorials to students struggling in a course and students having difficulties keeping up grades to maintain scholarship. Students who would like to avail of the tutorial service of the school can make an appointment through emails to their academic support personnel ${ }^{(3)}$. But even tutorial programs are offered; still the common approach to get a tutor is to personally go to the office of the student affairs or academic support personnel or to make an appointment provided online or through email and text messages. The option to get a list of tutors together with their expertise as well as feedback of these tutors from their previous tutee through performance ratings prior to getting the tutor has not yet been explored. Some schools also provide greater academic services to students by centralizing all tutors in one location with appointment based tutoring ${ }^{(4)}$.

Moreover, existing methods still raised questions such as whether or not it could be safe for both the tutor and tutee and none of the existing scheduling systems have addressed this concern ${ }^{(5)}$. In a private tutoring session, students' safety isn't assured at all times especially when sessions are being held outside of school premises with no proper monitoring of where the tutoring takes place ${ }^{(6)}$. Most tutorial appointment apps may provide good tutors but they still do not ensure a controlled environment that is constantly monitored by moderators.

Hence, this study is designed to develop GrabTutor, A Web and Mobile Application for Tutor Appointment System with GPS Security Feature that will provide a new rendezvous of tutors through which a tutee can select based on his/her preferences before setting an appointment for a face-to-face tutorial. GrabTutor will provide a list of available tutors with their expertise alongside with the performance ratings from previous tutees. Specifically, the study aims to (1) develop a mobile application for tutorial appointments that present the tutees ample information about the tutors to assist them in looking for appropriate tutor that would best fit their academic needs, (2) integrate location tracking system to pinpoint the tutorial session's location within the school premises, and (3) test and evaluate the proposed system in terms of usability and functionality.

\section{Materials and Methods}

\section{System Architecture}

The system architecture of the GrabTutor as shown in Figure 1, comprises of android supported smartphones, server and $\mathrm{Wi}-\mathrm{Fi} / 4 \mathrm{G} / 3 \mathrm{G}$ cell services for internet connectivity purposes. It shows that the android devices need internet to connect to the server in the cloud. There is also barcode for tutees and barcode scanner for tutors. Once scanned, it triggers the timer of the tutorial session and send GPS coordinates to the web application of the Administrator/Moderator for tracking and monitoring purposes.

\section{Tutor Appointment System Design}

\subsection{Process flow of the System}

The system has three (3) process flow. These are the Student Tutor Application Process, Student Tutor Application to Tutee's post, and Tutor and Tutee Tutorial Session.

2.1.1. Student Application Process. Figure 2 shows the application process of the student who wanted to apply as student tutor. The aspiring student will apply for tutorship to be filled out in the mobile application. The moderator will be notified once the student submits an application. The student will wait for the confirmation of the moderator for him/her to officially listed as tutor. 


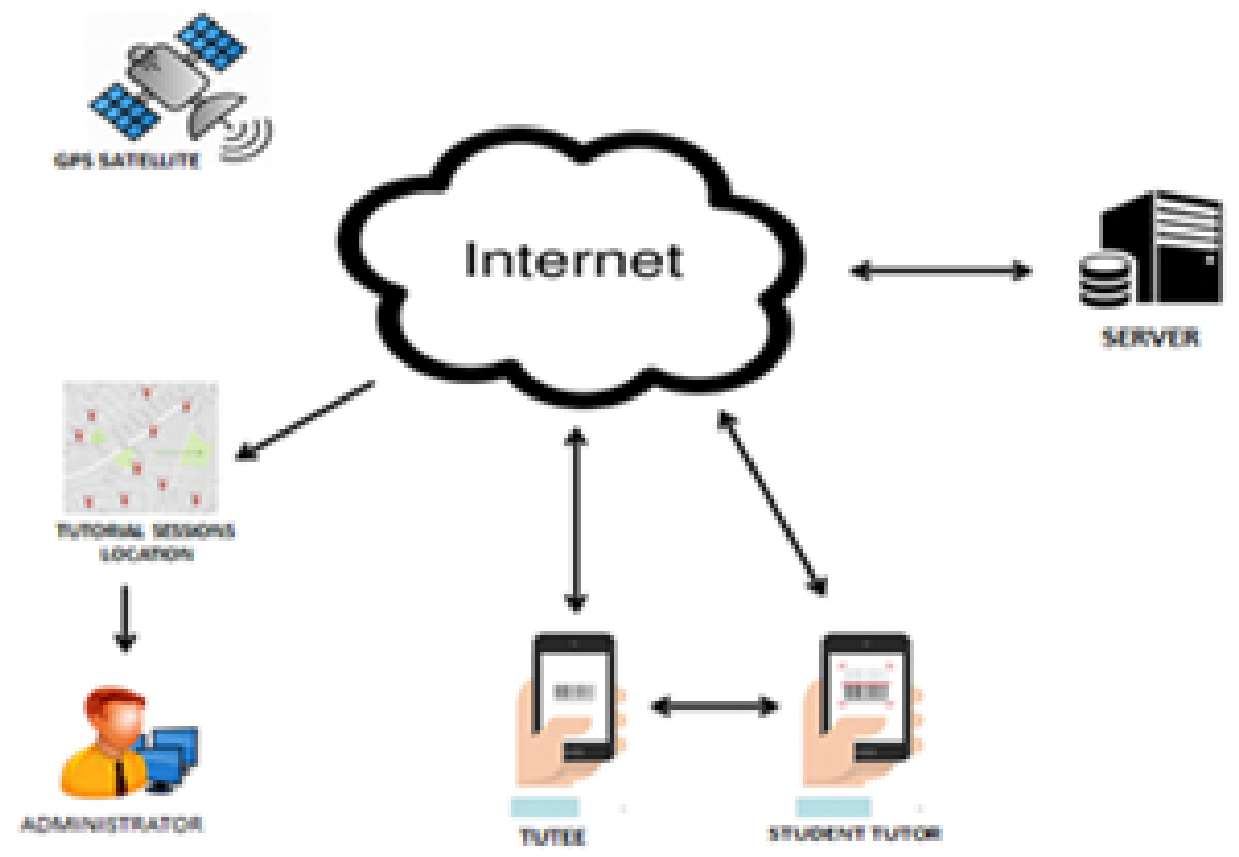

Fig 1. System Architecture of GrabTutor

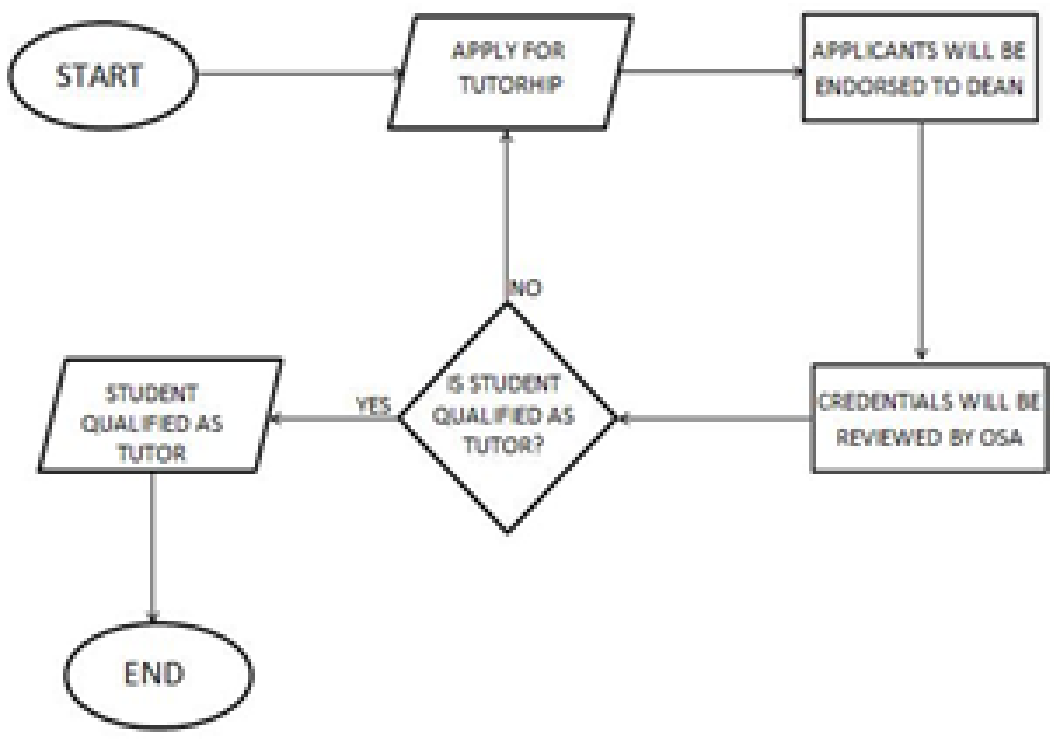

Fig 2. Student tutor application process 
2.1.2 Student Tutor Application to Tutee's Request. Figure 3 shows how the student tutor commits to tutee's post and be his tutor. The tutee fills out the form to set an appointment with tutor; then, he posts his/her appointment in order for tutors to view his appointment. She/he can view the recommended tutor in the subject he chose but he/she can't choose his/her tutor. Next, the tutor will view the post of tutee. He will then commit to accept the tutee in order to proceed to tutorial session

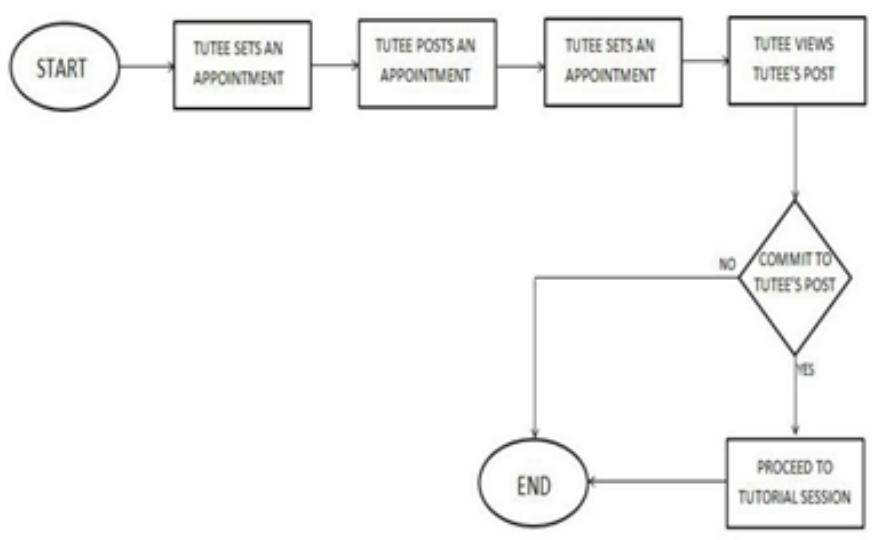

Fig 3. Student Tutor Applicationto Tutee's post

2.1.3 Tutor and Tutee Tutorial Session. Figure 4 shows the process of scanning the barcode to trigger the timer and the map marker. The tutor will turn on the GPS location to get the location of their device during session. After the tutor allows it, the tutor will then scan the barcode of the tutor in order to proceed to tutorial session. The timer will be triggered and the map marker of the tutor will be plotted in the map together with the relevant information.

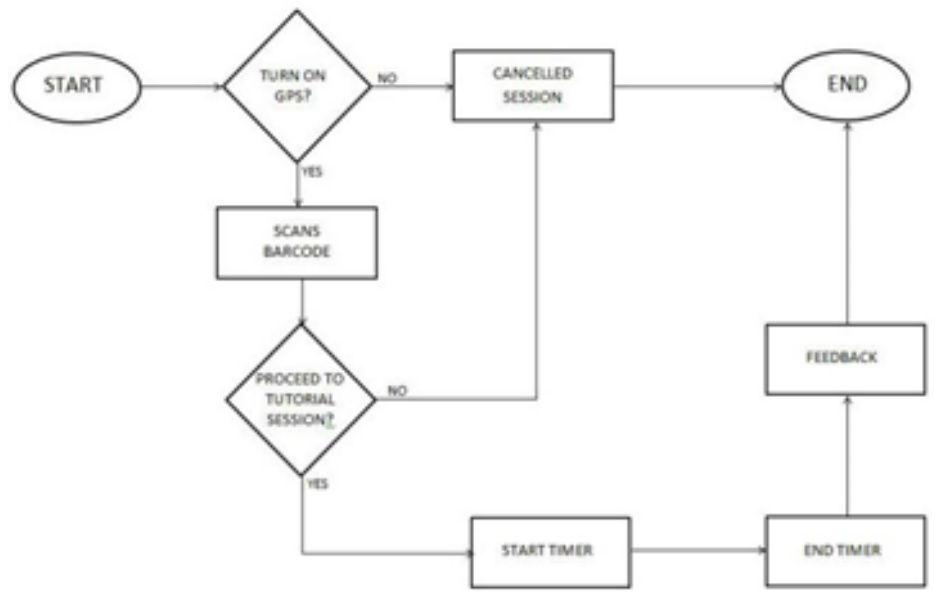

Fig 4. Tutor and Tutee TutorialSession

\subsection{Database Design}

Database design is the process of producing a detailed data model of a database. Figure 5 shows the database design of GrabTutor which contains 16 tables. The admin users table contains of the information of the moderators. The colleges table comprises the name of colleges in the school. This table used to connect to the subject area table. 
The primary key of the subject area table is SubjectAreaID. It is used to connect 2 tables namely: tutorials table and subject options. The tutorials table consists of the information of the tutee and the details of the tutorial. The committed tutorials table consists of the time and date of tutorial session, and the location (longitude and latitude) of the tutee in order to get the location of the tutor from the database that will be fetched by the web application to plot it in the map.
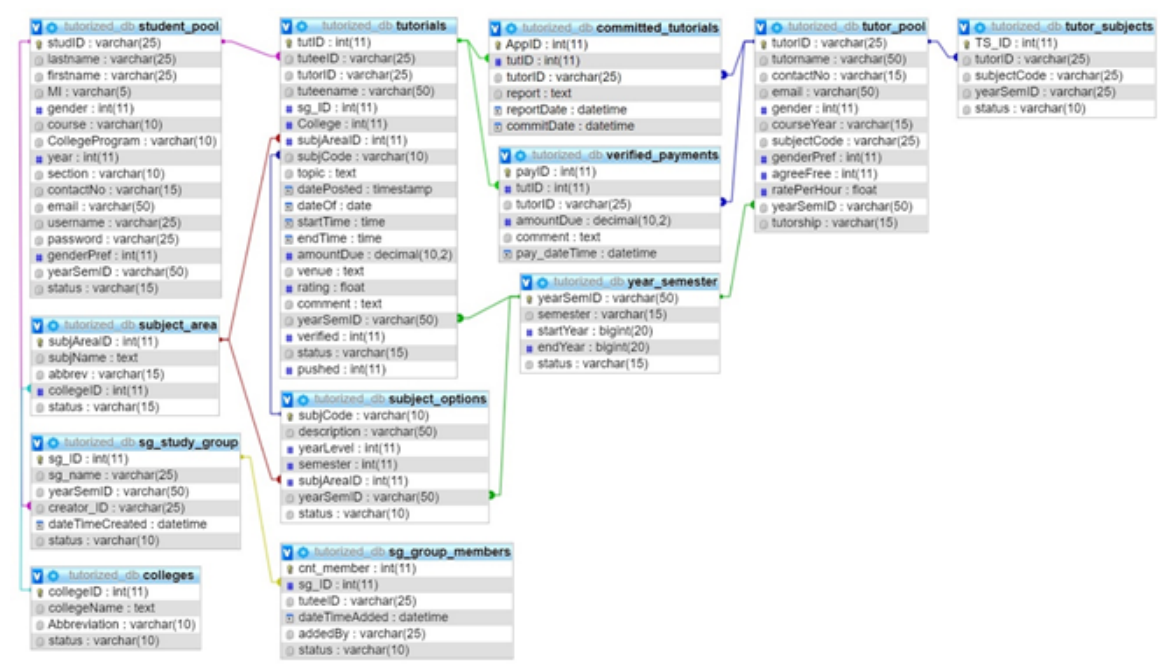

Fig 5. Database Design of GrabTutor

\section{Application Development}

\subsection{Mobile development}

In order for tutees to gain access to available tutors conveniently, a hybrid mobile was developed. It is an android application that connects to the database server hosted in the cloud. It was developed using Android SDK and Cordova. It presents a variety of feature for both tutees and tutors as an implementation of the three (3) process flow in Section 2.1.

Essentially, the mobile application is secured with an authentication mechanism through the login page as shown in Figure 6 (a). If an authorized user is granted access, it will be redirected to the main menu page as depicted in Figure 6(b), which allows the user to choose a role as either tutor or tutee. As a tutee, the user can request a tutor using the tutor finder page presented in Figure 6(c). It can set the necessary parameters and details of the tutorial that the user wants to request. Once the request has been posted, it will provide a list of recommended tutors accompanied by ratings given by previous tutees. On the other hand, the tutorial request is also visible to the tutors who are well qualified with the specifications of the tutee as reflected in Figure 6(d). As a Tutor, a certain user can apply for the tutorial as presented in Figure 6(e), which will remain in pending mode until the tutee, who is the requestor of the tutorial will accept the application as shown in Figure 6(f).If the tutee accepts the application, the tutorial session is officially booked and they will then meet face to face on the appointed date and time of the tutorial.

During the meet up, the tutor will need to scan the barcode in the mobile app of the tutee in order to trigger the timer, which indicates that the actual tutorial has started as shown in Figure 6(g) and Figure 6(h). Consequently, when the tutorial starts the location of the tutee and tutor will be sent to the database server, which will be accessed by the moderator's web application for monitoring. Once, the tutorial time expires the tutee will be prompted with a modal that asks to rate the tutor using a 5-star rating method and may also provide verbatim comments as depicted in Figure 6(i). 

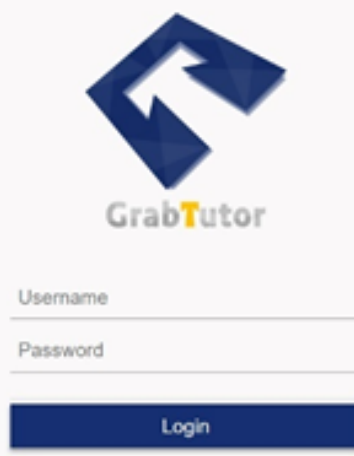

(a) Login Page

E Tutorial Requests

a Search for Tutorials

\section{Animation}

Variables

2018-00-17 | 8.00 Au-10-00 cosornoser: is rutus

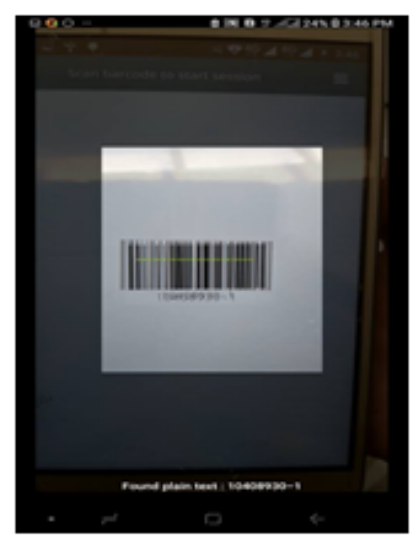

(g) Scan barcode

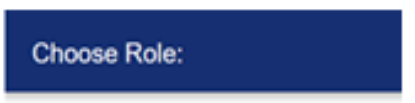

E Tulor Finder

(4) Set Parameters

Specoifc Topic

$1005 / 2018$

Find A Tutor

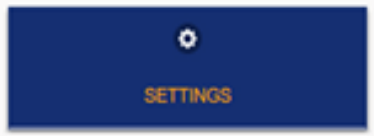

(b) Main Menu Page

\section{三 Tutorial Requests}

$\mathbf{x}$

3D Animation

Topic: Variables

Payment Verified: Yes

Your application will be forwarded to the tutee when

you apply.

\section{Apply}

(e) Apply for tutorial

\section{E Tuborial Requens}

\section{Session}

Start Nime: Wed Feb 272019 15:40:00 GMT +0800

End Time: Wed Feb 272019 15:50:00 GMT +0800

Time Left: 00:03:18

(h) Start tutorial timer
Post

(c) Tutor Finder Page

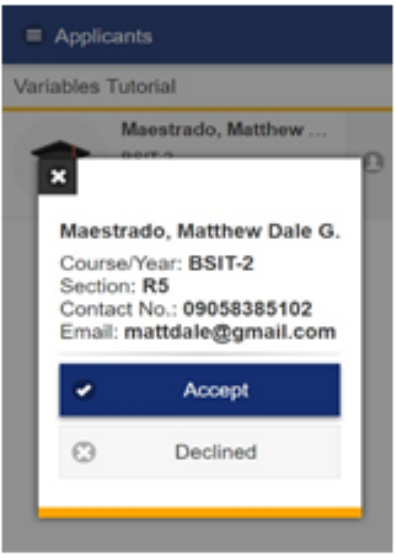

(f) Accept tutor modal

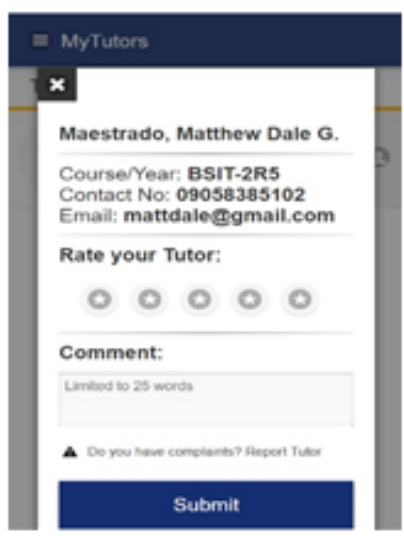

(i) Start tutorial timer

Fig 6. GrabTutor User Interfaces for Tutee and Tutor 


\subsection{Web development}

The mobile application is intended for the tutees and tutors. Meanwhile, the web application was created for the moderator of the system. It was developed using Code Igniter PHP Framework, JavaScript and Google Location API. It is granted with administrative access that allows the moderator to manage tutor and tutee applications and requests respectively. It primarily serves as a monitoring tool for tutor and tutee activities within the system and most importantly, keep track of their location as the tutorial happens.

In order to ensure that only the moderator can access the system, an authentication mechanism is also set in place as shown in Figure 7 (a). The moderator's primary responsibility is to screen tutor applications through the web application as depicted in Figure 7(b). Moreover, generation of reports and other administrative operations can also be performed by the moderator as presented in Figure 7(c).

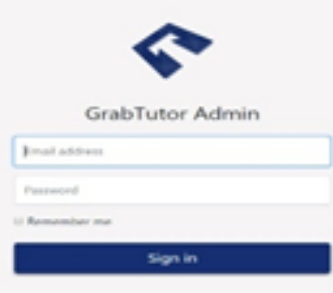

(a) Login Page

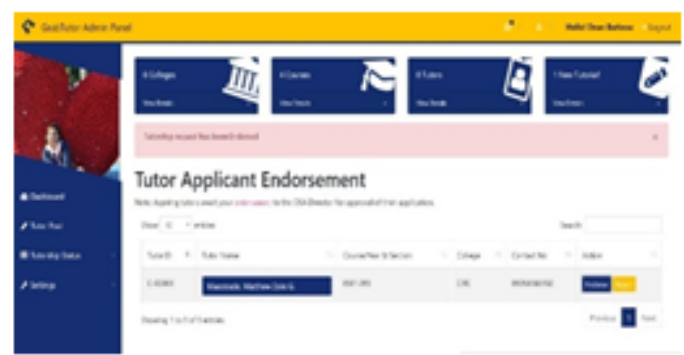

(b) Tutor Applicant Control Panel

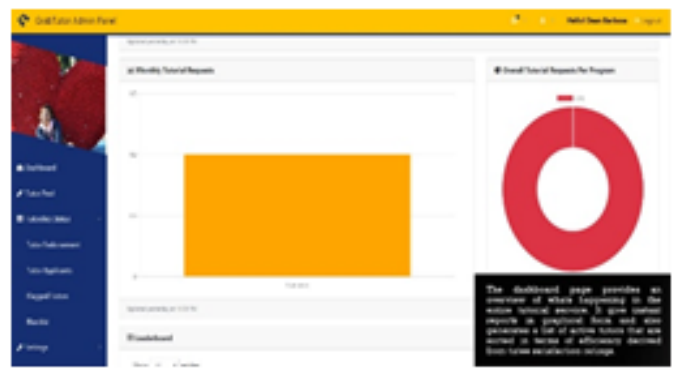

(c) Tutorial Requests Monitor

Fig 7. Web application user interfaces for the Moderator3

\subsubsection{GPS tracking of tutorial session}

GPS location tracking is a distinctive feature of this tutorial appointment system. Through the Google Location API, the web application has the ability to fetch the location of tutors and tutees inside a school campus. The GPS tracking is triggered when the barcode in the mobile application of the tutee is scanned by the tutor's mobile application which indicates that the actual face to face started already. The latitude and longitude of the current location of the tutorial is sent automatically to the mobile application. This feature adds a new dimension of security that would ensure students safety for the best interest of parents. Figure 8 shows the mechanism of plotting location in the map. While Figure 9 shows the implementation of GPS location tracking in the web application. 


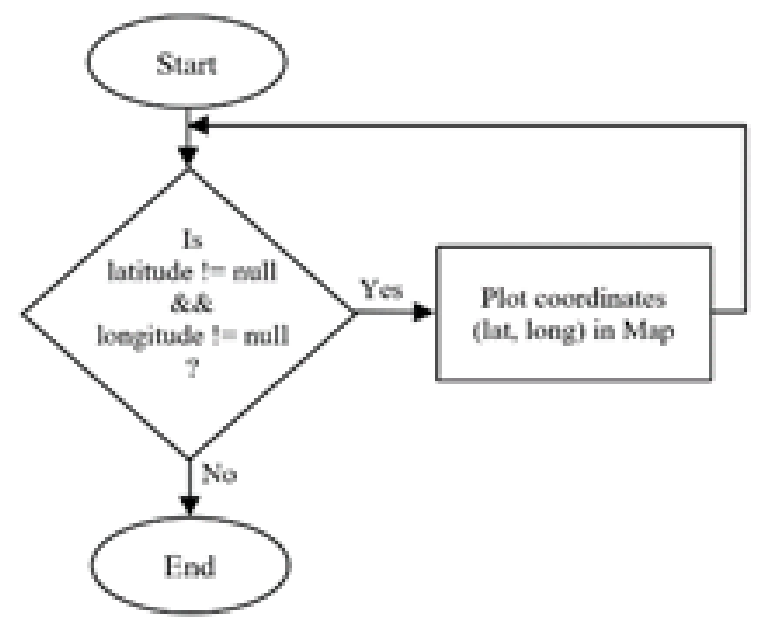

Fig 8. Location tracking flowchart

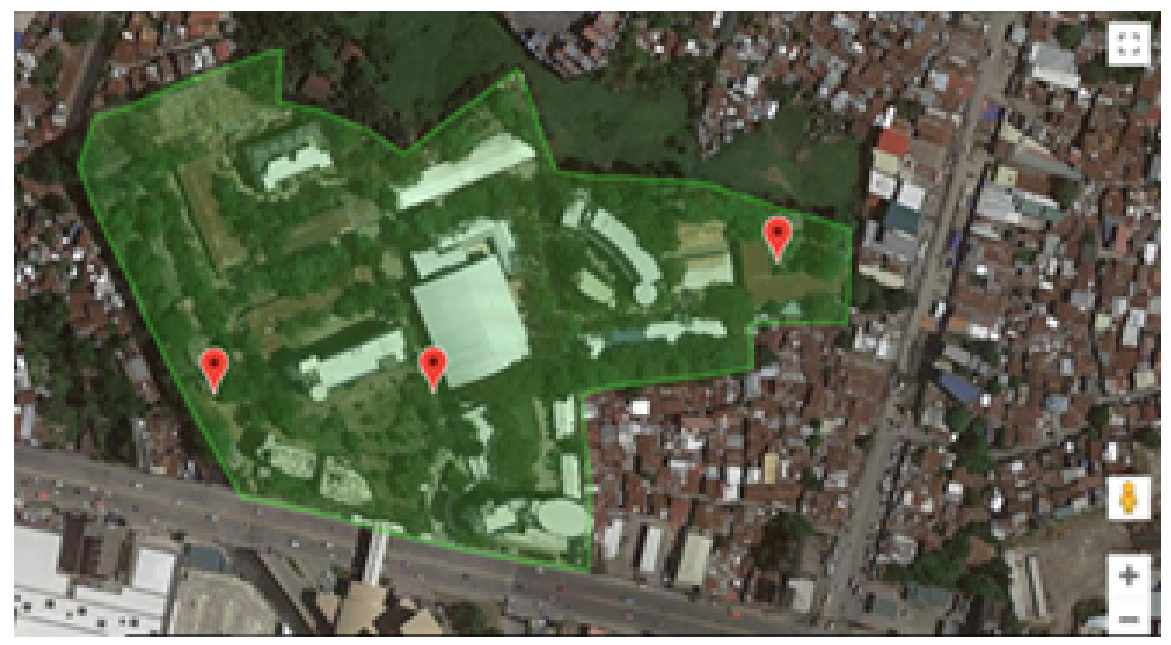

Fig 9. GrabTutor Web App Location Tracking

\section{Results and Discussion}

Figure 10 shows the usability test result of the mobile application. The respondents agreed that they like to use the application frequently and it is easy to use. The respondents agreed that they can use the application confidently. Meanwhile, Figure 11 shows the summary of the system usability of the web application. The respondents agreed that they like to use the application frequently and it is easy to use. The respondents agreed that they can use the application confidently.

Figure 12 shows the result of the functionality test of the system. All of the respondents agreed with a YES on functionality of the barcode scanning feature in the mobile application and the GPS location tracking feature in the web application. The integration of GPS technology in tutoring service adds more value in terms of the security of students involved in tutorials as not all schools have dedicated facilities for tutorial sessions. Moreover, unlike most tutor appointment applications such as GrabATutor, the proposed system is deployed as a mobile application which is more accessible to students nowadays without compromising the features available in its web application counterparts. 


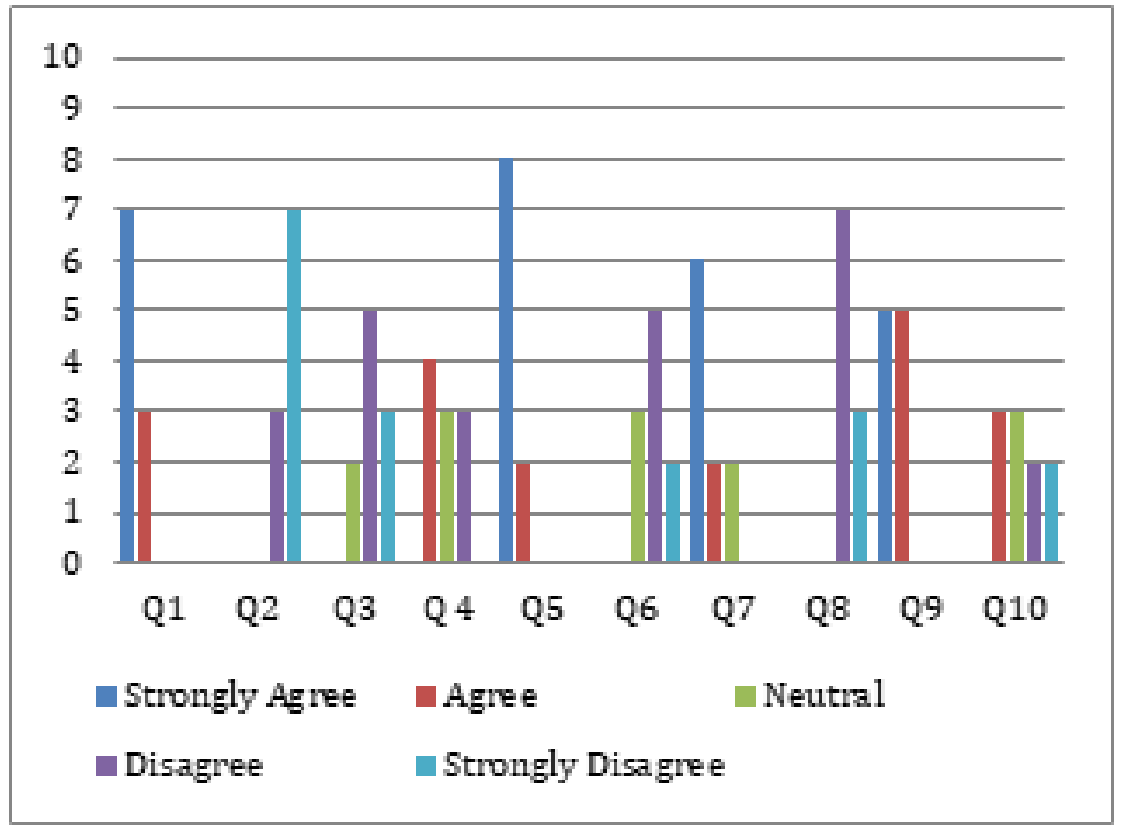

Fig 10. Mobile Application Usability Test Result

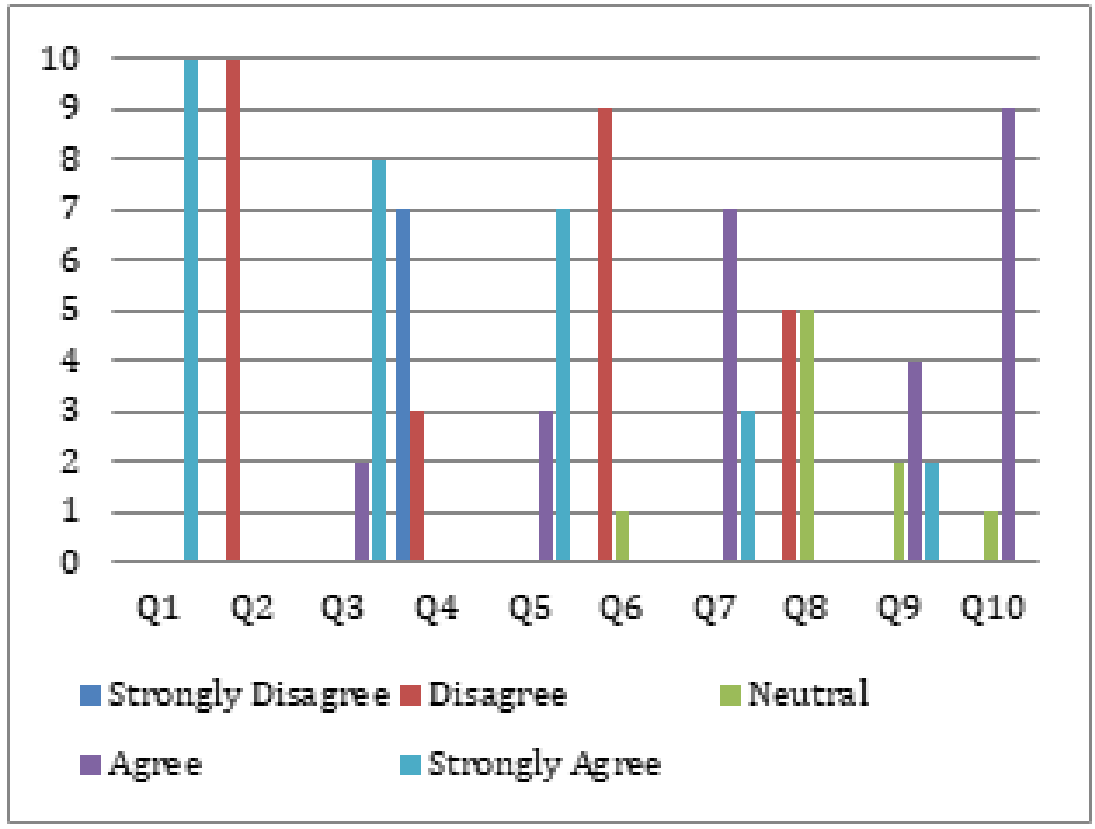

Fig 11. Web Application Usability Test Result 


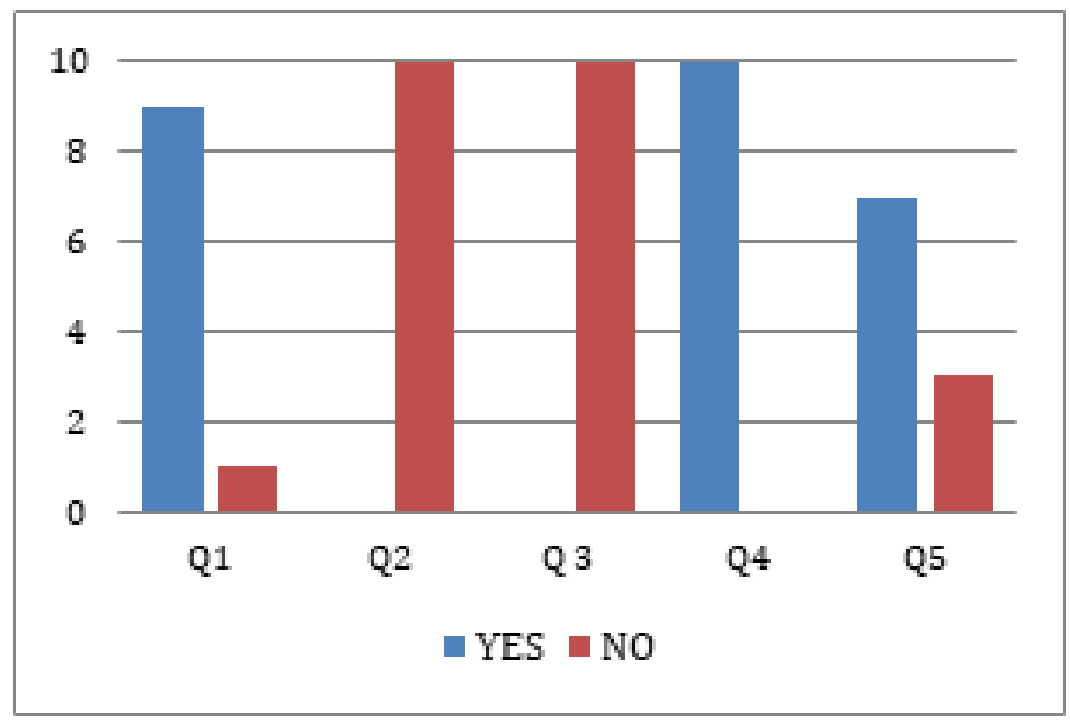

Fig 12. System Functionality Test Result

\section{Conclusions}

The system was developed to provide students with a tutorial booking mobile application with a GPS monitoring system that not only makes booking appointments with a tutor easier but also ensures safety of students both tutor and tutee. Based on the results of the evaluation of usability and functionality, the researchers conclude that the system makes it much easier for students to book an appointment with the mobile application by recommending the qualified tutors in an instant. The respondents also agree that the location tracking system could alleviate the risk of having to go somewhere else to meet up with a tutor.

\section{Acknowledgment}

The authors acknowledge the University of Science and Technology of Southern Philippines for providing the resources that needed for this project.

\section{References}

1) Peer Tutoring. . Available from: https:/odoc.princeton.edu/support/finding-help-academic-work/individual-peer-tutoring. Dateaccessed.

2) Cook PJ, Dodge K, Farkas G, Fryer RG, Guryan J, Ludwig J, et al. Not Too Late: Improving Academic Outcomes for Disadvantaged Youth. Evanston, IL. 2015. Available from: https://scholar.harvard.edu/files/fryer/files/not_too_late._improving_academic_outcomes_ for_disadvantaged_youth_2015.pdf.

3) M D, L C, B C, J F, R R, J S, et al. Dropout Prevention: A Practice Guide (NCEE 2008-4025). 2008. Available from: https://council-for-learning-disabilities.org/peer-tutoring-flexible-peer-mediated-strategy-that-involves-students-servingas-academic-tutors.Dateaccessed.

4) Johnson C. 2018. Available from: https://scholarworks.merrimack.edu/cgi/viewcontent.cgi?article=1028\&context=soe_studentpub.

5) Uddin MP, Nadim M, Imd, Afjal MI. GPS-based Location Tracking System via Android Device. International Journal of Research in Computer Engineering and Electronics. 2013;2.

6) Individual Peer Tutoring. . Available from: https://odoc.princeton.edu/support/finding-help-academic-work/individual-peer-tutoring. Dateaccessed. 